\title{
Embracing subjectivity in physics to support student empowerment
}

\author{
Andrea Wooley \\ Department of Physics and Astronomy, Western Washington University, Bellingham, WA, 98225, USA \\ Randeep Basara \\ Department of Engineering, Seattle University, 901 12th Ave, Seattle, WA, 98122, USA \\ Abigail Daane \\ Department of Physics, South Seattle College, $600016^{\text {th }}$ Ave. SW, Seattle, WA, 98106, USA
}

\begin{abstract}
Descriptions of the Nature of Science are in contrast with the widespread notion that "physics" is objective, unaffected by human influence. To better understand students' thinking about the nature of physics, we taught a lesson from the Underrepresentation Curriculum and collected written responses before and after in-class discussion about the nature of physics. In this manuscript, we share student responses about the presence of subjectivity in physics and relate them to commonly accepted ideas about the Nature of Science. Students tend to describe physics without reference to human influence. After discussing subjectivity, many students identify that while physical properties may be objective, human interpretation, biases, and values influence physics as well. Some students also consider our understanding of physics as incomplete. These discussions are a first step towards increasing awareness of structural and individual subjectivity. We believe this will ultimately support a more equitable, robust scientific community.
\end{abstract}

\footnotetext{
2021 PERC Proceedings edited by Bennett, Frank, and Vieyra; Peer-reviewed, doi.org/10.1119/perc.2021.pr.Wooley

Published by the American Association of Physics Teachers under a Creative Commons Attribution 4.0 license.

Further distribution must maintain the cover page and attribution to the article's authors.
} 


\section{INTRODUCTION}

Students' definitions of physics often reflect how society portrays the subject. For example:

"Physics is a math based science that describes real world phenomena. Does it matter who does it? Nope, anyone and everyone can do physics and should be encouraged to if they want to try. Physics isn't reliant on race, gender, or background, it's a universal subject." - Engineering Physics Student 3 (emphasis added)

Student 3 shares their perception that physics is separate from those who study it. We see this reflected in the teaching of physics: Euro-centric education does not emphasize the historical context and value multiple perspectives [1-2]. The stereotypical belief that physics is objective assumes physics to accurately reflect phenomena in the world without bias or cultural influence [3-5]. This prioritization of objectivity is a characteristic of white supremacy culture [6]. Our theoretical framework is shaped by the idea that to address systemic structures of oppression in physics, the subject cannot be viewed as entirely objective. To counter these assumptions of objectivity, we suggest that instructors make time for students to critically discuss the nature of science and the role of scientists. Scientific inquiry, a "complex social activity" [7], often involves working together to identify and avoid biases. Scientists blend logic and imagination to meet demands for evidence in particular contexts; discoveries emerge from interactions between power structures, individual physicists, and the physical world. Framing physics in this way celebrates the uniqueness of those who shape physics and empowers students to consider themselves valuable in the physics community.

Preliminary work on learning about the nature of physics at a private university found that after in-class discussion, students report shifts in thinking from physics as objective to physics as containing subjectivity [8]. Building on this work, we identify the specific ideas students share after a similar discussion in a different context. We highlight students' ideas that support previous research of students identifying subjectivity in physics post-discussion. We see that these ideas can support discussions of culture, race, gender, and diversity, which have received pushback in the past, [9-10] but can be powerful for identity development [11]. Acceptance of subjectivity in physics can empower students through their political relationship with physics [12], mobilizing them to work towards greater justice, changing the culture and field of physics [13].

\section{CONTEXT}

This study examines ideas of students in an introductory, calculus-based physics course for engineers and physicists at a minority-serving, two-year college, currently understudied [14]. The class was small and interactive, supporting community-building and active learning. The 26 students in this course were primarily engineering and computer science
TABLE I. Racial/gender demographics of students enrolled in second quarter physics courses.

\begin{tabular}{lc}
\hline \hline \multicolumn{1}{c}{ Demographic } & \% of Students \\
\hline Female & $21 \%$ \\
Male & $76 \%$ \\
Other Gender & $3 \%$ \\
\hline African American & $5 \%$ \\
Asian/Pacific Islander & $40 \%$ \\
Hispanic & $0 \%$ \\
Other Race/mixed & $11 \%$ \\
White & $37 \%$ \\
Not Reported & $6 \%$ \\
\hline \hline
\end{tabular}

majors. For anonymity, racial and gender demographics in Table 1 presents the physics student population over the entire 2017-2018 year instead of the single class from which data were collected. The instructor used elements of the Underrepresentation Curriculum (URC) [15] to assist students in critical discussions of the nature of science. The URC is a free, flexible curriculum for STEM instructors to teach about injustice and change the culture of STEM. Its development is an ongoing collaborative effort by multiple instructors over several years [e.g., 16-17]. The instructor implemented the URC in the middle of the second quarter of a yearlong introductory sequence. Students in the class knew each other well because of the interactive, collaborative class environment. For this study, we chose to analyze student ideas at the end of the first URC lesson entitled "Subjectivity and Objectivity in Science" [18] but before subsequent URC activities. In the following, we share a summary of the "Subjectivity and Objectivity in Science" lesson and recommend the reference above for a full explanation.

The purpose of the lesson was for learners to identify aspects of subjectivity present in physics that can explain the present inequity and systemic underrepresentation in the field. The lesson began by asking students to define physics, individually, then in small groups. Each group shared their definitions to the class. Based on those definitions, students decided whether they thought physics is subjective or objective. In this lesson, we define objectivity as facts uninfluenced by personal feelings or opinions. Subjectivity is defined as being influenced by personal ideas, opinions or feelings. The instructor then asked students to pair up with someone who made a different decision and listen to be convinced of others' ideas. The class came together again to discuss those ideas. Finally, each student wrote a reflection about their experience using the following prompt:

- What are you thinking about right now? and/or

- How are you feeling regarding our discussion?

Below, we share students' written reflections to gain insight into their ideas immediately following this particular lesson.

\section{METHODS}

Our qualitative content analysis sought to identify key ideas in student written responses that were collected before 
and after the lesson described above. All pre- and postresponses were redacted and assigned random numerical identifiers to maintain anonymity. All three authors cocreated emergent categories through an iterative cycle of identifying and refining key themes [19]. We initially coded the data individually, compared our results, and discussed discrepancies. After discussion, we simplified our initial nine categories into three key themes. Finally, we compared students' ideas to generally accepted ideas about the nature of science found in Science for All Americans [7], the American Association for the Advancement of Science publication about a vision for science literacy, upon which the national science standards are based.

All three authors have intimate associations with this lesson and data. The third author designed and taught the lesson. The first and second authors participated in this lesson as students in previous courses. Their experiences of the lesson differed: they each bring their own perspectives and insight to this research. We view these experiences as bringing additional expertise, in the form of memberchecking [20-21], that supports a more rigorous understanding of student responses. That said student responses were possibly truncated because of end-of-class time constraints. Thus, ideas are not representative of all student ideas, even within this particular group. Our interpretation of responses inevitably uses prior experiences and expectations about how students have previously responded. This study is also likely influenced by the authors' personal goals to improve physics culture. For this reason, we made extra effort to bracket our ideas as standalone, separate from our analysis [21].

\section{RESULTS: STUDENT IDEAS OF SUBJECTIVITY \& OBJECTIVITY}

At the end of the discussion (described in the context above), 21 of 26 students recorded their ideas about whether physics is subjective or objective. It seems important to note that the majority of students $(16 / 21)$ articulate that the physical properties of the universe do not depend on how we study them. Students include descriptions of physics as "how things work," universal or physical "laws and properties," and "theories" in their writing. We find this description of the physical properties in all categories, regardless of their conclusion about the presence of subjectivity in physics.

$7 / 21$ students who responded to the post-discussion reflection prompt, maintain that physics is objective. 14/21 students acknowledge some aspects of subjectivity in physics. Five students do not specify their opinion of physics as subjective or objective and instead write about their experience with the discussion.

We organized the remaining student responses into three categories: (A) students describe physics as objective, (B) students describe physics as incomplete (i.e., much is left unknown), and (C) students describe physics as including subjectivity. Responses in (B) used "incompleteness" as an example to support either physics as objective or subjective.

\section{A. Students describe physics as objective}

While the majority of students' pre-responses include a description of physics as objective laws, a few students $(4 / 21)$ do not mention any possible subjectivity. Below is an excerpt from Student 13 that embodies this idea:

"[Physics is] objective because Physics is the study of how things interact in the universe (bounded by calculations and laws of physics). Subjective leans more towards psychology $\rightarrow$ feelings and thoughts."

Student 13 contrasts physics, "bounded by calculations and laws," with psychology that they associate with "feelings and thoughts." Their distinction between a scientific discipline based in human emotion (psychology) and a discipline that is "objective" (physics) may imply that subjectivity does not factor into the field of physics.

\section{B. Students describe physics as incomplete and either objective or subjective}

Six students described physics as incomplete. Three students use this idea to conclude that physics is objective and three others use this same reasoning to conclude that physics is subjective. Student 23's reflection states that physics is objective, and also acknowledges current limits of human understanding:

"I thought physics is objective, because even though we have many ideas about physics, there is an absolute truth of what physics is and just because we may not know it or have theories right now, doesn't change the absolute truth about it."

Student 23 describes the "absolute truth" of physical phenomena as evidence of objectivity. They differentiate our "many ideas about physics" from the "truth of what physics is". By writing, "we may not know it or have theories right now," they imply that the limit of our theories is flexible; as technology and human ability improves, our understanding gets closer to the truth. Other students corroborate that the "many ideas" people have do not impact the objectivity of physics. For example, Student 9 acknowledges that "nonphysics" people may not believe the laws of physics: "it depends on the ideas of what people think if it is true or not." Student 14 states, "fools sometimes make it [physics] subjective." They write that people's beliefs or lack of knowledge lead to an incomplete understanding.

In contrast, a few other students use the incompleteness of our understanding as evidence for subjectivity. For example, student 19 describes the uncertainty of physics theories as evidence of physics' subjective nature:

"I agree with the fan of subjective why they think about it. For example, in the space, there are many theories how our universe is going on after big bang. Some of them if shrink or extend or stable. But, I think there is an answer whatever it is. Just we don't know now." 
In this quote, physics has "many theories" for understanding phenomena, such as the creation of the universe. They write, "we don't know now" what the answer is and use this to explain why physics is subjective. Other students' writing aligns with this idea that physics is incomplete and therefore subjective. For example, Student 4 describes physics as "the best we can do with what we know", in this context, "best" implies that better understanding might be out there.

\section{Students describe physics as including subjectivity}

In reflections, 14/21 students acknowledge subjectivity explicitly or by describing subjective aspects of physics (one response may appear in multiple subcategories). We highlight student examples of how humans introduce subjectivity through interpretation (C.1), bias (C.2), or values (C.3).

\section{Human interpretation introduces subjectivity}

Some students acknowledge subjectivity in physics by describing the influence of human interpretation on our understanding. Their reflections describe physical phenomena as an objective truth, separate from the human study of those phenomena that they define as subjective. For example, Student 18 writes:

"Philosophy is cool. I feel like physics can be both objective and subjective. There [are] answers out in the universe of physics and that is objective. It then becomes subjective when people start to study it. But does that make physics itself subjective?",

Responses in this theme question what counts as physics. Student 18 considers the subjectivity or objectivity of physics to be a philosophical question. They write that physics answers "out in the universe" are objective, and then identify subjectivity in physics arising from human involvement. Student 3 poses a question, "is it our interpretation of those [physical] laws and properties that make up the world?...if it is, then physics is subjective." Students, who acknowledge that physics involves both the world around us and us, identify subjectivity in physics due to the nature of "our interpretation." These ideas align with the goals of scientists who "strive to make sense of observations of phenomena by constructing explanations for them that use, or are consistent with, currently accepted scientific principles" [7].

Several students describe the presence of subjectivity in physics resulting from interpretation. For example:

"...physics is objective because it's based on observed things, but then it's what about the theory parts of physics, it's someone's understanding of how they interpreted something they observed and the person could be wrong. If and when they are proved wrong, doesn't that make physics subjective? Physics therefore must be subjective and objective." (Student 16)

Initially, Student 16 describes physics as objective because it is "based on observed things." Then they describe how theories/interpretations based on someone's understanding of observations "could be wrong." They use the idea that a theory is an assumption, produced by human cognition, as evidence that subjectivity is present in physics.

\section{Human bias introduces subjectivity}

Student 25 describes bias as evidence for subjectivity. "After our discussion I still believe that physics is both subjective and objective. There is only one answer to questions and problems, that's why it's objective. On the other hand, humans are always going to have biases. We're not machines."

This idea complements Student 16's ideas about incorrect interpretation and also describes a specific reason for our incorrect knowledge: "humans are always going to have biases." The presence of bias broadens the scope of subjectivity from simply making mistakes to a more systemic and universal influence across physics.

Bias, (mis)interpretation, and the study of the physical world are generally acknowledged as examples of human influence in science: "Scientific evidence can be biased in how the data are interpreted, in the recording or reporting of the data, or even in the choice of what data to consider in the first place." [7] The choice of "recording and reporting" data includes scientists' use of representation of concepts. What scientists choose to highlight or neglect in models affects what others learn from the data. Student 22 builds on Student 3's ideas and also describes subjectivity that arises through choice of representation:

"I think the fundamentals of the universe are objective...However, I think the way we represent and interpret that law/laws is subjective as there is subjectivity in everything. Even the math we use to describe phenomena is arbitrary."

This response mirrors previous ideas that physical phenomena are objective and that the study of physics is subjective because of "the way we represent and interpret" those phenomena. They build on those ideas by using math as evidence of subjectivity. Physicists use/create mathematical representations based on interpretations and this student writes that our use of math is "arbitrary" possibly meaning that it is determined by preference and bias.

\section{Human values introduces subjectivity}

Another way students identify subjectivity manifesting in physics is in our interpretation of what is worth studying. Student 8 describes subjectivity arising from our choice to study certain subjects over others. "I'm thinking about how physics means differently to learners. The reason they want to learn physics will shape their ideas about physics." The "reason" a learner is motivated to understand physics will "shape" the knowledge that they retain and apply. This idea aligns with generally accepted beliefs in the scientific community: "Scientists' nationality, sex, ethnic origin, age, political convictions, and so on may incline them to look for 
or emphasize one or another kind of evidence or interpretation" [7]. Students reference the personal interests of scientific learners as directing the focus of their ideas. Student 10 concludes that the presence of subjectivity depends "on the thing we solve." We interpret this as recognition that one particular topic in physics might have more subjectivity than another. Alternatively, this student might be indicating that subjectivity appears when we decide to solve certain problems over others. Student 20 articulates that even when we all focus on the same problem to solve, "everybody has different thoughts about physics. ...Everybody has different ways to approach a problem." These "different thoughts" and "ways to approach" physics are also examples of choices a scientist must make that influence the direction of learning and research. Student 20's ideas mirror aspects of this widely accepted description:

"Scientists differ greatly from one another in what phenomena they investigate and in how they go about their work; in the reliance they place on historical data or on experimental findings and on qualitative or quantitative methods" [7]

\section{DISCUSSION AND IMPLICATIONS}

The written reflections from students presented in Section IV.A indicate that ideas of physics as an objective entity are strong: one third of students maintained the perspective that physics is objective, and most students described physics as simply the universe and laws, which dictate physical phenomena. In Section IV.B, students articulate that our understanding of universal truths are incomplete, acknowledging our limitations. Finally, in Section IV.C, 14/21 students readily identify that while physical properties (e.g., laws) may be objective, human involvement creates subjectivity. Student reflections include myriad ways that subjectivity appears in physics: through human bias, values, and interpretation.

After analysis of these students' ideas about subjectivity, the third author has since adjusted the lesson to include more focus on certain aspects of science that did not initially emerge. For example, students in this class did not discuss systems of power (and oppression) in the physics community that dictate access, barriers, funding, opportunities, support, and sense of belonging [7]. Instructors can pay attention to what is NOT brought up in class and make sure to include certain topics for discussion such as the potential benefits and/or drawbacks of these practices and norms (e.g., funding, hiring, promoting, peer-review, competition to publish first, emphasis on the written word as most powerful, rewarding individual successes over community efforts, mentorship, apprenticeship, etc.).

With all of the required standards to teach in the introductory physics curriculum, it may be hard to imagine devoting class time to discuss the nature of physics. And yet, including this conversation in introductory (and more advanced) physics courses can produce several benefits that extend beyond the lesson itself. First, we see these responses as a productive step in students' efforts to articulate their understanding of the nature of physics. Students consider how humans impact the physics they study, and in doing so, practice identifying how personal experience, values, and reasoning can affect the culture and progress of physics. This encourages opportunities for diverse thinking and learning. Students may feel empowered to express themselves more authentically in learning experiences and seek out alternative ideas and methods from their peers. Students may more readily bring up limitations and biases that will support their future investigative and practical choices in labs and research and in other sciences [22]. Students may also reference examples of subjectivity such as structural bias and barriers when they discuss their career choices. Finally, these conversations open the door for additional learning about both the consequential and moral justifications for diversity and equity in the field using additional URC lessons [15]. They also support the identification of systemic barriers that make it more challenging for some people to participate [23]. Productive discussions can shift students' ideas about their ability to impact physics culture [8] and empower students to produce ideas for changing said culture [24].

\section{CONCLUSIONS}

Our goal is ultimately to provide students the space to recognize the study of physics as a human endeavor influenced by the wants and needs of society, with standards and norms defined by those in power and a product of the intellect and bias of those who study it. Our research showed that discussions about the nature of science support students in describing physics as incomplete and subjective. Future work can investigate whether the improvements recommended in the discussion section expand the range of ideas shared by students post-discussion.

Students need the opportunity to discuss the problematic assumption that physics is objective and to consider how human impact on research is reflected in curricula and textbooks. We therefore recommend teaching the URC in every physics class as part of the general curriculum. Talking about subjectivity during physics classes gives us all (students and instructors alike) the chance to situate physics within confines of our cultural norms, expectations, and power structures so they can feel empowered to make changes to their classes and the larger community [23-24].

\section{ACKNOWLEDGMENTS}

We are grateful to E. Schoene, J. Ashcraft, L. Villarta, C. Gosling, D. Doucette, B. Geller, D. Dounas-Frazer, and the URC editors for their support. This material is based upon work supported by the National Science Foundation under grant no. S-STEM1643580. 
[1] G.L. Cochran, S. Hyater-Adams, C. Alvarado, C. PrescodWeinstein, and A.R. Daane, Social Justice and Physics Education. in Teaching and Learning for Social Justice and Equity in Higher Education, 2021, edited by C. C. Ozaki, L. Parson (Palgrave Macmillan, Cham., 2021).

https://doi.org/10.1007/978-3-030-69947-5_7

[2] J. Hatton, and P. B. Plouffe, Science and its ways of knowing (Prentice Hall, 1997).

[3] S. Traweek, Beamtimes and lifetimes (Harvard Univ. Press, Boston, 2009).

[4] A. J. Gonsalves, Cult. Stud. Sci. Educ. 9(2), (2014).

[5] A. Bug, "Has feminism changed physics?", Signs: Journal of women in culture and society 28(3), (2003).

[6] K. Jones, and T. Okun, "Dismantling racism: A workbook for social change groups," dRworks. https://www.dismantlingracism.org/. Retrieved 4/15/2021.

[7] F. J. Rutherford, and A. Ahlgren, Science for All Americans, (Oxford University Press, 1991).

[8] S. R. Decker, and A. R. Daane, "Teaching about Inequity: Shifts in Student Views about Diversity in Physics," in AIP Conf. Proc. p. 108-111. (2018). https://www.compadre.org/per/items/detail.cfm?ID=14580

[9] A. C. Johnson, Sci. Educ. 91(5), (2007).

[10] A. R. Daane, and V. Sawtelle, "Student discourse about equity in an introductory college physics course," in AIP Conf. Proc. p. 88-91. (2016). 10.1119/perc.2016.pr.017

[11] R. M. Lock and Z. Hazari, "Discussing underrepresentation as a means to facilitating female students' physics identity development," Phys. Rev. Phys. Educ. Res. 12, 020101 (2016).

[12] A. S. Dimick, "Student empowerment in an environmental science classroom: Toward a framework for social justice science education," Sci. Educ., 96, 990, (2012).
[13] K. Mitchell-Polka, "The physics classroom as a space for empowerment" in AIP Conf. Proc. (2020). https://www.compadre.org/per/perc/2020/Detail.cfm?id=81 $\underline{55}$

[14] S. Kanim and X.C. Cid, "Demographics of physics education research," Phys. Rev. Phys. Educ. Res. 16, 020106 - Published 27 July 2020

[15] The Underrepresentation Curriculum, https://www.underrep.com. Retrieved 4/15/2021.

[16] M. Rifkin, "Addressing underrepresentation: Physics teaching for all," Phys. Teach. 54, 72 (Feb. 2016).

[17] A.R. Daane, S.R. Decker, and V. Sawtelle, "Teaching about Racial Equity in Introductory Physics Courses," Phys. Teach. 55(328). (2017).

[18] "Subjectivity in Science Lesson" http://www.underrep.com. Retrieved 4/15/2021.

[19] K. Krippendorff, Content Analysis: An Introduction to Its Methodology (Sage, CA, 2013), 3rd edn.

[20] A.G. Candela, Exploring the Function of Member Checking. The Qualitative Report, 24(3), 619-628. (2019). Retrieved from https://nsuworks.nova.edu/tqr/vol24/iss3/14

[21] J. W. Creswell, Qualitative Inquiry and Research Design: Choosing Among Five Approaches. India: SAGE Publications. (2012).

[22] M.M. Stein, E.M Smith, and N.G. Holmes, Confirming what we know: Understanding questionable research practices in intro physics labs in AIP Conf. Proc. (2018). https://www.compadre.org/per/items/detail.cfm?ID=14856

[23] S. Herrera, I.A. Mohamed, A.R. Daane, "Physics from an underrepresented lens: What I wish others knew" Phys. Teach., 58(5) p. 294-296, (2020).

[24] A. A. Arielle, K. S. Bretl, A. Ross, and A.R. Daane, "Introductory physics students' insights for improving physics culture," in AIP Conf. Proc. (2019). 